\title{
Identification of diagnostic upper gastrointestinal cancer tissue type-specific urinary biomarkers
}

\author{
HOLGER HUSI ${ }^{1-3}$, MARCO FERNANDES $^{2}$, RICHARD J. SKIPWORTH $^{3}$, JANICE MILLER ${ }^{3}$, \\ ANDREW D. CRONSHAW ${ }^{4}$, KENNETH C. H. FEARON ${ }^{3}$ and JAMES A. ROSS ${ }^{3}$

\footnotetext{
${ }^{1}$ Department of Diabetes and Cardiovascular Science, University of the Highlands and Islands, Inverness IV2 3JH;

${ }^{2}$ BHF Glasgow Cardiovascular Research Centre, University of Glasgow, Glasgow G12 8TA; ${ }^{3}$ School of Clinical Sciences and Community Health; ${ }^{4}$ School of Biological Sciences, University of Edinburgh, Edinburgh EH16 4SB, UK
}

Received September 11, 2018; Accepted January 2, 2019

DOI: $10.3892 /$ br.2019.1190

\begin{abstract}
Several potential urinary biomarkers exhibiting an association with upper gastrointestinal tumour growth have been previously identified, of which S100A6, S100A9, rabenosyn-5 and programmed cell death 6-interacting protein (PDCD6IP) were further validated and found to be upregulated in malignant tumours. The cancer cohort from our previous study was subclassified to assess whether distinct molecular markers can be identified for each individual cancer type using a similar approach. Urine samples from patients with cancers of the stomach, oesophagus, oesophagogastric junction or pancreas were analysed by surface-enhanced laser desorption/ionization-time-of-flight mass spectrometry using both CM10 and IMAC30 ( $\mathrm{Cu}^{2+}$-complexed $)$ chip types and LC-MS/MS-based mass spectrometry after chromatographic enrichment. This was followed by protein identification, pattern matching and validation by western blotting. We found $8 \mathrm{~m} / \mathrm{z}$ peaks with statistical significance for the four cancer types investigated, of which m/z 2447 and 2577 were identified by pattern matching as fragments of cathepsin-B (CTSB) and cystatin-B (CSTB); both molecules are indicative of pancreatic cancer. Additionally, we observed a potential association of upregulated $\alpha$-1-antichymotrypsin with pancreatic and gastric cancers, of PDCD6IP, vitelline membrane outer layer protein 1 homolog (VMO1) and triosephosphate isomerase (TPI1) with oesophagogastric junctional cancers, and of complement $\mathrm{C} 4-\mathrm{A}$, prostatic acid phosphatase, azurocidin and histone-H1
\end{abstract}

Correspondence to: Dr Holger Husi, Department of Diabetes and Cardiovascular Science, University of the Highlands and Islands, Office H/107, Centre for Health Science, Old Perth Road, Inverness IV2 3JH, UK

E-mail: holger.husi@uhi.ac.uk

Abbreviations: emPAI, exponentially modified protein abundance index; GI, gastrointestinal; OGJ, oesophagogastric junction

Key words: SELDI-TOF, mass spectrometry, pancreatic cancer, upper gastrointestinal cancer, urine biomarker with oesophageal cancer. Furthermore, the potential pancreatic cancer biomarkers CSTB and CTSB were validated independently by western blotting. Therefore, the present study identified two new potential urinary biomarkers that appear to be associated with pancreatic cancer. This may provide a simple, non-invasive screening test for use in the clinical setting.

\section{Introduction}

A significant number of cancer-related deaths worldwide are associated with malignant tumours of the upper gastrointestinal (GI) tract, such as the stomach, oesophagus, oesophagogastric junction (OGJ) (1) and pancreas. The Global Burden of Disease study undertaken in 2015 identified these types of cancer to have a very poor prognosis, with gastric and oesophageal cancers contributing to 10.3 and $5.4 \%$, respectively, of all cancer deaths globally, with both exhibiting a male preponderance (2). Pancreatic cancer is associated with a dismal 5-year survival rate of $3 \%$ and its incidence appears to be increasing annually $(3,4)$. This has led to the development of various therapeutic strategies to prolong survival, which have had limited success, including improved surgical techniques, anti-angiogenesis therapies and adjuvant/neoadjuvant chemoradiotherapy $(5,6)$. Pancreatic cancer cases in particular are usually diagnosed at an advanced stage and, therefore, the possibility of a non-invasive intervention to halt tumour progression is greatly reduced. Therefore, it is crucial to devise methodologies to not only pharmacologically treat, but also to diagnose this type of cancer at an early stage.

The most successful and widely used cancer assays to date are based on the detection and quantification of glycans in the serum using antibodies against CA19.9 (7) or CA125 (8), a pan-cancer marker that targets carbohydrate-associated epitopes of immunoglobulin heavy chains (9). CA19.9, with a reported sensitivity of $90-100 \%$ and specificity of $70-98 \%$ for pancreatic cancer detection (10), is the best biomarker assay currently in clinical use. However, the positive predictive value of CA19.9 for detecting pancreatic cancer is only $0.9 \%$ in the asymptomatic population due to a dependency of blood-group markers where CA19.9 can be used (11). It is also associated with biliary obstruction (12) and has been proven unable to distinguish pancreatic cancer from matched controls in larger studies, highlighting its poor clinical utility as a tumour marker (13). 
Other potential cancer protein biomarkers have been identified by antibody arrays, such as H2B histone using the IPO-38 antibody to define gastric cancer (14), M2 pyruvate kinase in faeces as a GI cancer marker (15) and tumour-associated trypsin inhibitor as a marker of liver metastasis and colorectal cancer (16), and by mass spectrometry, such as S100A9 for upper GI cancer (17), as well as S100A6 for a wide array of tissue cancers, including thyroid (18), gastric (19), ovarian (20), hepatocellular (21), bowel (22), breast (23) and upper GI (17) cancers.

Numerous potential biomarkers were also identified by gene array screens of diseased tissue (24-27) and by microRNA array screens of circulating biofluids (28), in the most frequent cancers affecting the GI tract $(29,30)$. The latter is considered as a promising source of clinically relevant biomarkers, when compared with tissue gene array screens, since the use of biopsies as a predictive diagnostic tool remains unrealistic for use in the clinical setting. Additionally, prediction of several cancer types based on serum metabolic profiles is also feasible (31). Concomitantly, collaborative efforts have been made to promote the detection of plasma-derived metabolite markers for pancreatic cancer diagnosis $(32,33)$.

There is a clear need to simplify the medium to be screened due to genetic variations and population heterogeneity in order to define reliable disease markers. A substantially less complex system, such as the urine, which contains $\sim 5,000$ proteins (34), would be a preferred medium to screen for protein or peptide biomarkers. This has a number of advantages, including non-invasive sampling for patients, ease of sampling, and unrestricted availability under normal conditions (35). Urine itself is also relatively stable in terms of protein and peptide composition and fragmentation state compared with other body fluids, such as the serum, where proteolytic degradation by endogenous proteases has been shown to occur during or after sample collection (36).

In the present study, surface-enhanced laser desorption/ionization-time-of-flight mass spectrometry (SELDI-TOF-MS) was used to screen human urine samples from patients with upper GI tumours to establish biomarker patterns using the CM10 and IMAC30 chip types, as well as independent LC-MS/MS mass spectrometry screening and a combined bioinformatics data analysis, in order to present a potentially useful approach to diagnosing upper GI tissue type-specific cancers in humans using novel potential biomarkers.

\section{Materials and methods}

Materials. All buffers, gels and SELDI chips were purchased from Bio-Rad Laboratories Ltd. (Hemel Hempstead, UK), and all other chemicals were obtained from Sigma-Aldrich; Merck $\mathrm{KGaA}$ (Gillingham, UK), unless stated otherwise in the text.

Sample collection. Urine samples were obtained from 83 patients with upper GI cancers undergoing potentially curative resection. The participant demographics are summarised in Table I and provided in detail in Table SI. The participants' age ranged between 43 and 83 years. Fasting urine samples were obtained at induction of anaesthesia. One-third of the patients had pancreatic tumours, one-third had oesophageal cancer, approximately one-sixth had malignancies of the OGJ and one-sixth suffered from gastric cancer. All procedures were approved by the local research ethics committee and written informed consent was obtained from the patients. The study conformed to the standards set by the Declaration of Helsinki. All urine samples were stored at $-40^{\circ} \mathrm{C}$. Long-term storage of samples $\left(>1\right.$ month) was at $-80^{\circ} \mathrm{C}$.

SELDI-TOF-MS. SELDI chips (CM10 and IMAC30) were prepared for sample application according to the manufacturer's recommendations. Briefly, IMAC30 chips were loaded with $0.1 \mathrm{M} \mathrm{CuSO}_{4}$, washed with water, neutralised with $0.1 \mathrm{M}$ $\mathrm{NaHAc}(\mathrm{pH}$ 4.0) and again washed with water, followed by two washes with $0.1 \mathrm{M} \mathrm{NaHPO}_{4}$ and $0.5 \mathrm{M} \mathrm{NaCl}$; CM10 chips were washed twice with $0.1 \mathrm{M} \mathrm{NaHPO}_{4}$ (pH 4.0). All chips were processed in a bioprocessor assembly by incubating $0.1 \mathrm{ml}$ urine and $0.1 \mathrm{ml}$ binding buffer [IMAC30: $0.1 \mathrm{M}$ $\mathrm{NaHPO}_{4}, 0.5 \mathrm{M} \mathrm{NaCl}$; CM10: $0.1 \mathrm{M} \mathrm{NaHPO}_{4}(\mathrm{pH} 4.0)$ ] for $1 \mathrm{~h}$ at room temperature with vigorous shaking, followed by three washes with $0.2 \mathrm{ml}$ binding buffer for $5 \mathrm{~min}$ each at room temperature with vigorous shaking and two washes with $0.2 \mathrm{ml}$ water at room temperature with vigorous shaking. All chips were removed from the bioprocessor assembly, air-dried and $1 \mu \mathrm{l}$ energy-absorbing matrix [a saturated solution of sinapinic acid in 50\% acetonitrile (ACN) and $0.5 \%$ trifluoroacetic acid] was added twice. Air-dried chips were analysed in a PCS4000 SELDI-TOF instrument (Bio-Rad Laboratories, Ltd.) by measuring the 1,000-25,000 Da range with a low laser setting of $2.5 \mu \mathrm{J}$, and spectra were exported as '. $\mathrm{xml}$ ' files. The SELDI instrument was calibrated using the ProteinChip All-In-one peptide standard (Bio-Rad Laboratories, Ltd.). The source voltage was $25,000 \mathrm{~V}$ and the detector voltage was $2,946 \mathrm{~V}$. Quality control and consistency were ensured by using one random pool of urine samples on one spot per chip each. Spectra of the full analysis were recorded in two large batches to minimize instrument variability and drift. Spectral alignments of all quality controls ensured consistency of all spectra.

SELDI-TOF-MS data processing. ProteinChip Data Manager Software version 4.1 with integrated Biomarker Wizard cluster analysis (Bio-Rad Laboratories, Ltd.) was used for analysis. SELDI-TOF-MS traces were split into the four cancer type groups. The baseline was subtracted from individual $\mathrm{m} / \mathrm{z}$ traces and the profiles were normalised using total ion current, followed by identification of peak clusters using the cluster analysis tool. Peaks were selected in the first pass with a signal-to-noise $(\mathrm{S} / \mathrm{N})$ ratio of $>5$ and a valley depth of at least 3 , and in the second pass with a $\mathrm{S} / \mathrm{N}$ of 2 and a valley depth of 2 . The cluster mass window was set to $0.2 \%$ of the mass. Clustered peaks were only included if they occurred in at least $10 \%$ of all spectra. The resulting P-values, mean and median $\mathrm{m} / \mathrm{z}$ values, and the intensities of the clustered peaks were exported and saved as '.csv' files. A two-sample t-test was used to compare mean normalized intensities between the groups. The P-value was set at 0.05 to indicate statistically significant differences. Clustered peak lists were analysed with the Biomarker Pattern Software (Bio-Rad Laboratories, Ltd.) and $\mathrm{m} / \mathrm{z}$ vs. intensity matrices were analysed using decision tree analysis, selecting the 
Table I. Demographics of the cohort used in this study $(n=83)$.

\begin{tabular}{|c|c|c|c|c|c|}
\hline Cancer type ${ }^{a}$ & Oesophagus & Pancreas & OGJ & Gastric & Total \\
\hline No. of patients & 27 & 28 & 13 & 15 & 83 \\
\hline Mean age ${ }^{\mathrm{b}}$ (years) & $66.5(10.8)$ & $63.5(9.1)$ & $61.5(8.1)$ & $70(7)$ & $65.3(9.5)$ \\
\hline Male & 22 & 16 & 12 & 9 & 59 \\
\hline Female & 5 & 12 & 1 & 6 & 24 \\
\hline
\end{tabular}

${ }^{a}$ Based on the histological assessment of each tumour type. ${ }^{b}$ Mean age of the participants according to the various groupings (standard deviation). Sex breakdown (male/female) and the total sample numbers (total) of the groups is included as number of participants. OGJ, oesophagogastric junction.

standard error rule of minimum-cost tree regardless of size, and using the Gini method. V-fold testing was set to 1,000. All samples from one cancer type were used to build exploratory networks by using all other cancer samples as 'negative' controls. Tree model building was performed using a selected $\mathrm{m} / \mathrm{z}$ peak as the only deciding factor to obtain sensitivity and specificity values. Sensitivity was defined as the probability of predicting specific cancer cases, and specificity was defined as the probability of predicting other cancers.

Peak isolation and identification by LC-MS/MS and Mascot searching. Peaks observed in the CM10 and IMAC30 chip types (Table SIII) that exhibited marked expression differences between tissue-specific cancer samples, and statistical significance in the cluster analyses with $\mathrm{P}<0.05$, were further investigated. Urine $(0.5 \mathrm{ml})$ from positive or negative samples in relation to specific peaks was added to $30 \mu \mathrm{l} \mathrm{CM10}$ or IMAC30 $\left(\mathrm{Cu}^{2+}\right.$-complexed $)$ spin column resin (Bio-Rad Laboratories, Ltd.) and $0.75 \mathrm{ml}$ binding buffer [0.1 $\mathrm{M} \mathrm{NaHPO}_{4}(\mathrm{pH} 4.0)$ for CM10 resins, and $0.1 \mathrm{M}$ $\mathrm{NaHPO}_{4}(\mathrm{pH} 7.0)$ including $0.5 \mathrm{M} \mathrm{NaCl}$ for IMAC30 resins] and incubated for $1 \mathrm{~h}$ at room temperature under constant agitation. Unbound material was removed and the resin was washed four times with $0.3 \mathrm{ml}$ binding buffer. Bound material was separated by electrophoresis on a $16.5 \%$ Tris-Tricine gel (Bio-Rad Laboratories, Ltd.), and gel bands in the region of 2-10 kDa were excised following Coomassie staining (BioSafe Coomassie; Bio-Rad Laboratories, Ltd.). Positive and negative samples were both selected on the presence and absence of a specific $\mathrm{m} / \mathrm{z}$ peak to be identified based on SELDI-TOF-MS analysis. Proteins and peptides from gel bands were digested in situ with trypsin, the resulting peptides eluted with ACN, and analysed by LC-MS/MS as described previously (17). Data-dependent acquisition was controlled by Xcalibur software and fragmentation spectra were then processed by Xcalibur and BioWorks software (Thermo Fisher Scientific, Inc., Loughborough, UK) and submitted to the Mascot search engine (Matrix Science, London, UK) using UniProt/SwissProt (release July 2010, Homo sapiens, 18055 sequences) as the reference database. The Mascot search parameters were as follows: Enzyme specificity, trypsin; maximum missed cleavage, 1; fixed modifications, cysteine carbamidomethylation; variable modification, methionine oxidation; precursor mass tolerance, $\pm 3 \mathrm{Da}$; and fragment ion mass tolerance, $\pm 0.4 \mathrm{Da}$. Only Mascot hits with a false discovery rate of $<0.05$ were taken into consideration.
Mascot-SELDI matrix matching. Observed proteins with at least two peptide matches from the LC-MS/MS analysis were then further analysed by pattern matching based on SELDI-TOF-MS measured expression levels of peaks of interest (expected abundance in selected samples). This was performed using software written in-house, which compares observed protein expression patterns in a pre-defined set of samples (LC-MS/MS results) against a matrix of peak patterns (SELDI-TOF clustered peak intensities, where estimated peaks are set to null) in the same set of samples. The scoring is based on sensitivity (percent observed over expected) and specificity (percent not observed over not expected), and the results are presented in descending order of cumulative scores.

Western blotting and validation of Mascot search results. Cross-validation of identified peaks was performed by western blotting of raw urine samples for anti-cathepsin B (CTSB) and anti-cystatin B (CSTB) blots (20 $\mu$ l per sample) using standard protocols (37). The antibodies used were rabbit anti-human CTSB (G-60; 1:1,000; cat. no. 3373; Cell Signaling Technology, Danvers, MA, USA), rabbit anti-human serum albumin (1:1,000; cat.no.A3293; Sigma-Aldrich; Merck KGaA), mouse anti-human CSTB (1:400; cat. no. sc-101510; Santa Cruz Biotechnology, Santa Cruz, CA, USA), and the peroxidase-coupled secondary antibodies were from Upstate (Lake Placid, NY, USA), used at a dilution of 1:5,000. Detection of signals was performed by chemiluminescence using ECL western blotting reagents (Thermo Fisher Scientific, Inc., Cramlington, UK).

\section{Results}

Urinary screening via SELDI-TOF-MS analysis. Urine samples from 83 patients diagnosed with various types of upper GI cancer were analysed in the course of this study (Table I). A full demographic with additional clinical data is provided in Table SI. The mean age of the participants was 65 years, $34 \%$ of the cohort suffered from pancreatic cancer, $33 \%$ from oesophageal cancer, $18 \%$ from gastric tumours and $15 \%$ from cancer of the OGJ. SELDI-TOF-MS analysis was selected to screen the samples mentioned above based on our previous study, where we described global specific markers for upper GI cancer (20). We found that both the metal chelator resin IMAC30 $\left(\mathrm{Cu}^{2+}\right.$-chelated $)$ and the weak cation exchanger CM10 yielded the best and most reproducible results. All samples were measured by SELDI-TOF-MS to obtain a peak pattern of identified molecular constituents, allowing us to stratify tissue type-specific cancers. We selected to compare only samples 
from cancer patients against each other to circumvent the issue of accidentally tracking lead markers that may be associated with additional underlying conditions, such as inflammatory responses, which are commonly observed in cancer patients.

SELDI analysis of the CM10 chip type-based screen of the 83 cancer urine samples resulted in 9,379 peaks, and the IMAC30 chip analysis provided a cumulative peak list of 3,346 features (Table SII). Clustering of observed peaks using the thresholds described in Materials and methods resulted in 328 cluster peaks for the CM10 chips, and the IMAC30 chip type gave yield to 92 common peak clusters above the set thresholds (Table SIII). Both analyses were performed by omitting estimated peaks in order to restrict and raise the specificity of potential marker peaks and, therefore, only included well-defined and separated individual peaks from all spectra. Statistical analysis revealed that 8 peak clusters are potentially associated with the various cancer types, namely $\mathrm{m} / \mathrm{z} 2444$ and 2557 for pancreatic cancer in the IMAC30 set, and $\mathrm{m} / \mathrm{z} 2447$ and 9618 in the CM10 chip-based set for the same cancer type, $\mathrm{m} / \mathrm{z} 5511$ and 4908 for OGJ cancer and m/z 4639 for gastric cancer in the IMAC30 chips, and $\mathrm{m} / \mathrm{z} 4141$ for oesophageal cancer in the CM10 chip set (Fig. 1; Table II). All potential markers have $\mathrm{P}<0.05$ and exhibit upregulation in the associated disease. It is noteworthy to mention that any given peak cluster can comprise more than one molecular entities. Therefore, it is possible that both the 2444 and $2447 \mathrm{~m} / \mathrm{z}$ peaks share an overlap in their molecular constituents; however, they include other peptides or proteins which are unique in this specific peak cluster based on the sensitivity and specificity distribution. The frequency distribution analysis of those 8 peaks shows that the $\mathrm{m} / \mathrm{z} 9618$ cluster from the CM10 chip screen displays the best stratifier for pancreatic cancer (Fig. 1D), with a sensitivity of $61 \%$ and a specificity of $82 \%$ in upper GI cancer cases. Comparison of all 8 peaks in non-cancer control cases measured in our previous study (17) also demonstrated that the $\mathrm{m} / \mathrm{z} 9618$ peak cluster exhibits the same frequency distribution in both non-cancer and non-pancreatic cancer cases of $\sim 30 \%$, whereas this frequency is doubled in pancreatic cancer for this peak cluster. The best predictor, based on a low frequency in non-cancer cases, was the $\mathrm{m} / \mathrm{z} 2577$ cluster in pancreatic cancer (Fig. 1B), with a $79 \%$ frequency in this cancer type, a $62 \%$ frequency in other cancers, and a $10 \%$ occurrence in non-cancer patient urine, with a sensitivity of $86 \%$ and a specificity of $44 \%$. The elevated amount of the observed $\mathrm{m} / \mathrm{z} 4141$ peak cluster associated with oesophageal cancer (Fig. 1H) demonstrates the best specificity of $100 \%$, but exhibits a relatively poor sensitivity of only $19 \%$ due to the relatively low frequency of one in four samples where this peak can be measured. Low frequency values were also observed for the OGJ cancer-associated elevated peak cluster at $\mathrm{m} / \mathrm{z} 4908$ (Fig. 1F), with good sensitivity and specificity values of $92 \%$ and $66 \%$, respectively. A more pronounced potential OGJ marker in terms of quantitative changes was measured at m/z 5511 (Fig. 1E) with a high specificity (94\%), but a relatively low sensitivity (38\%).

We then endeavoured to identify the molecular identity of the $\mathrm{m} / \mathrm{z} 2447$ peak, since it showed the highest sensitivity of $100 \%$ in the detection of pancreatic cancer. This was performed by selecting 8 samples, of which 4 either contained the cluster peak at $\mathrm{m} / \mathrm{z} 2447$, or did not show the peak

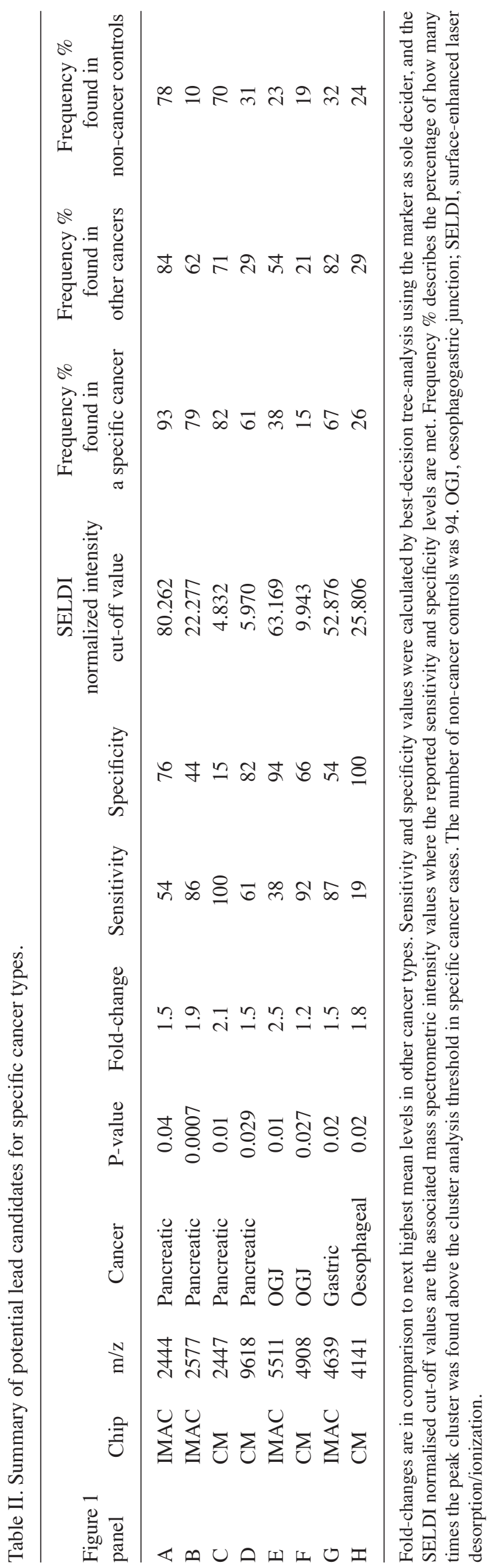


A

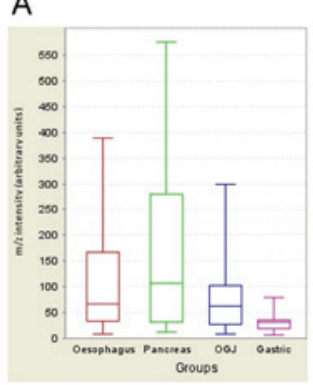

$\mathrm{E}$

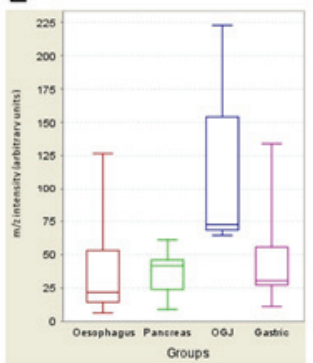

B

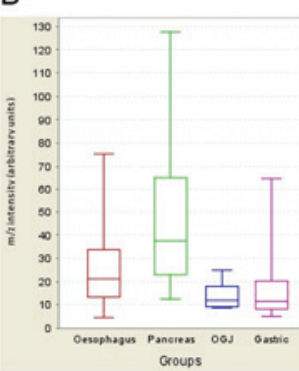

$\mathrm{F}$

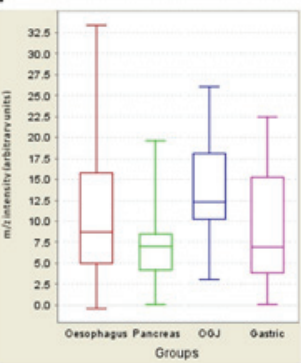

C

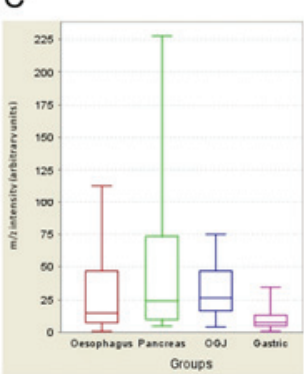

G

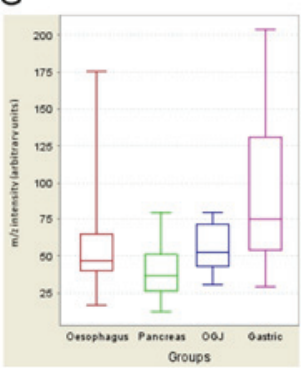

D

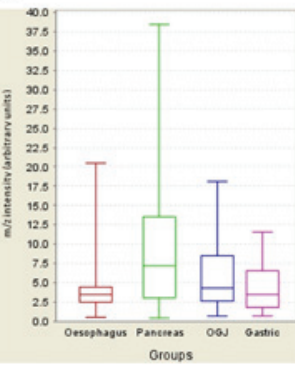

$\mathrm{H}$

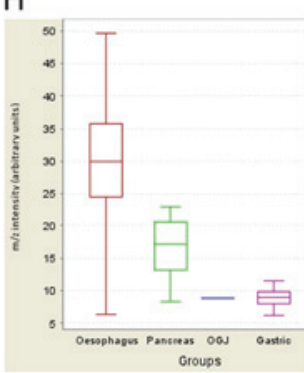

Figure 1. Expression profiling of potential biomarkers. The expression profiles of six potential markers specific for the four cancer types are displayed as whisker plots (median, lower and upper quartile and upper and lower extreme values). The x-axis contains the categorical variable representing four cancer types [oesophageal (red), pancreas (green), OGJ (blue) and gastric (pink)], while the $\mathrm{y}$-axis shows the normalised $\mathrm{m} / \mathrm{z}$ intensity (arbitrary units). [(A) (m/z 2444 ), (B) (m/z 2577), (E) (m/z 5511), (G) (m/z 4639)] are derived from the IMAC30 chip type, and [(C) (m/z 2447), (D) (m/z 9618), (F) (m/z 4908), (H) (m/z 4141)] from the CM10 chip type (see Table II for m/z and fold-change descriptions for panels A-H). (A-D) Potential pancreatic tumour markers; (E and F) OGJ marker; (G) gastric cancer marker; (H) oesophageal cancer marker. OGJ, oesophagogastric junction.

according to the SELDI-TOF scans. The samples were individually batch-absorbed on CM resin; bands in the $2-4 \mathrm{kDa}$ range were excised after peptide gel electrophoresis and processed as described previously (17). The molecular cluster of interest was shown to consist of fragments from CTSB, $\alpha$-1-antichymotrypsin precursor and immunoglobulin $\gamma$ and $\kappa$ chains (Table III). The same result was obtained using an independent approach, as described below.

We then analysed the molecular constituents of human cancer patient urine in the $2-10 \mathrm{kDa}$ molecular weight range using chromatographic protein and peptide enrichment on CM10 and IMAC30 resins, followed by gel separation, trypsin digestion and LC-MS/MS fragmentation, as before. Samples were selected based on the SELDI-TOF analysis results to either contain the $8 \mathrm{~m} / \mathrm{z}$ peaks of interest (50\% of samples per subset) or not in subsets of groups of 16 samples each. This resulted in the analysis of 145 urine samples, including repeats, from 62 upper GI cancer patients, of which 42 unique patient urines were enriched on CM10, and 40 unique patient samples on IMAC30 chromatography resins. 950 non-redundant proteins were identified in the CM10 resin-based approach by Mascot searching, and 600 unique proteins could be observed in the IMAC30-based enrichment, totalling 1,228 unique and non-redundant proteins in the combined datasets (Table SIV). Protein expression pattern matching (i.e., molecules identified by LC-MS/MS and Mascot searching, and matching to peak expression patterns observed by SELDI-MS) was performed by using an automated computer program written in-house based on the filtered Mascot dataset, where each individual identification was based on Mascot scores $>16$ and consisting of at least 2 peptides each. All proteins were identified in at least three independent samples. Table III lists all found by this approach, including a list of publications describing the relevance of these molecules in tumour growth. A fully detailed list of these molecules, including peptide sequences, is supplied in Table $\mathrm{SV}$.

The $\mathrm{m} / \mathrm{z} 2444$ peak observed in pancreatic cancer using the IMAC30 chromatography resin, as well as the pancreatic cancer-associated $\mathrm{m} / \mathrm{z} 2447$ peak in the CM10 dataset, matched the expression pattern of $\alpha$-1-antichymotrypsin (SERPINA3 or AACT). The same molecule, although a different proteolytic fragment of $\mathrm{m} / \mathrm{z} 4639$, could also be a potential biomarker for gastric cancer. A fragment of CSTB matched the observed pancreatic marker of $\mathrm{m} / \mathrm{z} 2577$ in the IMAC30 resin type-derived dataset, and other potential pancreatic markers of m/z 2477 and 9618 matched the presence of peptides from immunoglobulins. The $\mathrm{m} / \mathrm{z} 2477$ cluster distribution pattern also coincides with the measured expression pattern of CTSB.

Potential biomarkers for OGJ tumours include programmed cell death 6-interacting protein (PDCD6IP), matching the observed pattern of $\mathrm{m} / \mathrm{z} 5511$ in the IMAC30 resin dataset, vitelline membrane outer layer protein 1 homolog (VMO1) and triosephosphate isomerase (TPI1), which matched the $\mathrm{m} / \mathrm{z} 4908$ peak pattern in SELDI-TOF-MS measurements. The $\mathrm{m} / \mathrm{z} 4141$ clustered peak in the CM10 enriched dataset, which may be indicative of oesophageal cancer, matched the LC-MS/MS observed expression pattern of several molecules, namely complement $\mathrm{C} 4-\mathrm{A}(\mathrm{C} 4 \mathrm{~A})$, prostatic acid phosphatase (ACPP), azurocidin (AZU1) and histone H1.2, H1.3 or H1.4.

Both CTSB and CSTB were also cross-validated by western blot analysis (Fig. 2), and both molecules exhibited a very good correlation to the expected cluster-peak pattern observed by SELDI analysis, thereby validating both the predictor model and the Mascot identification, as well as the SELDI peak clustering of these molecules, and may represent viable prognostic biomarkers for pancreatic cancer. 


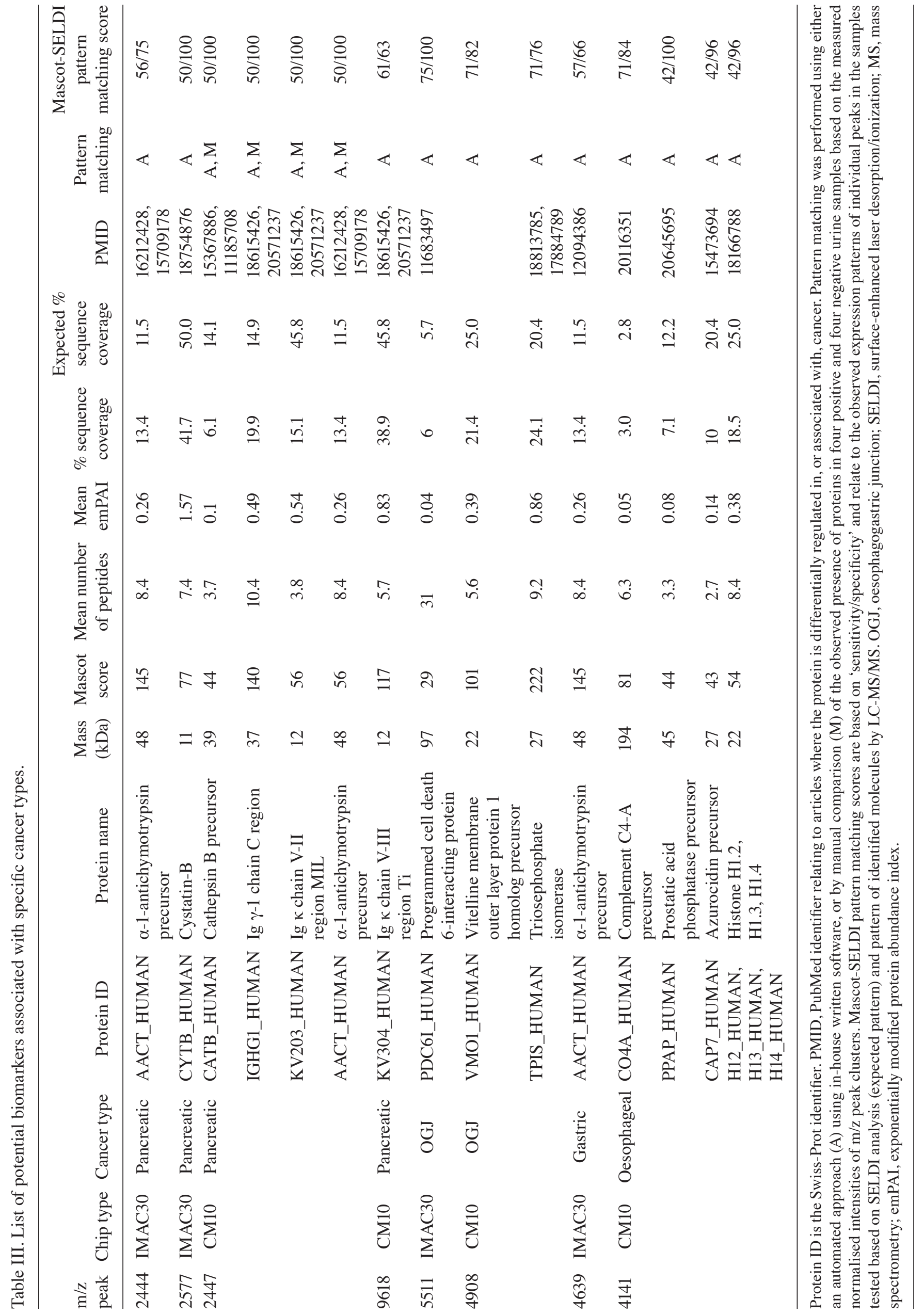



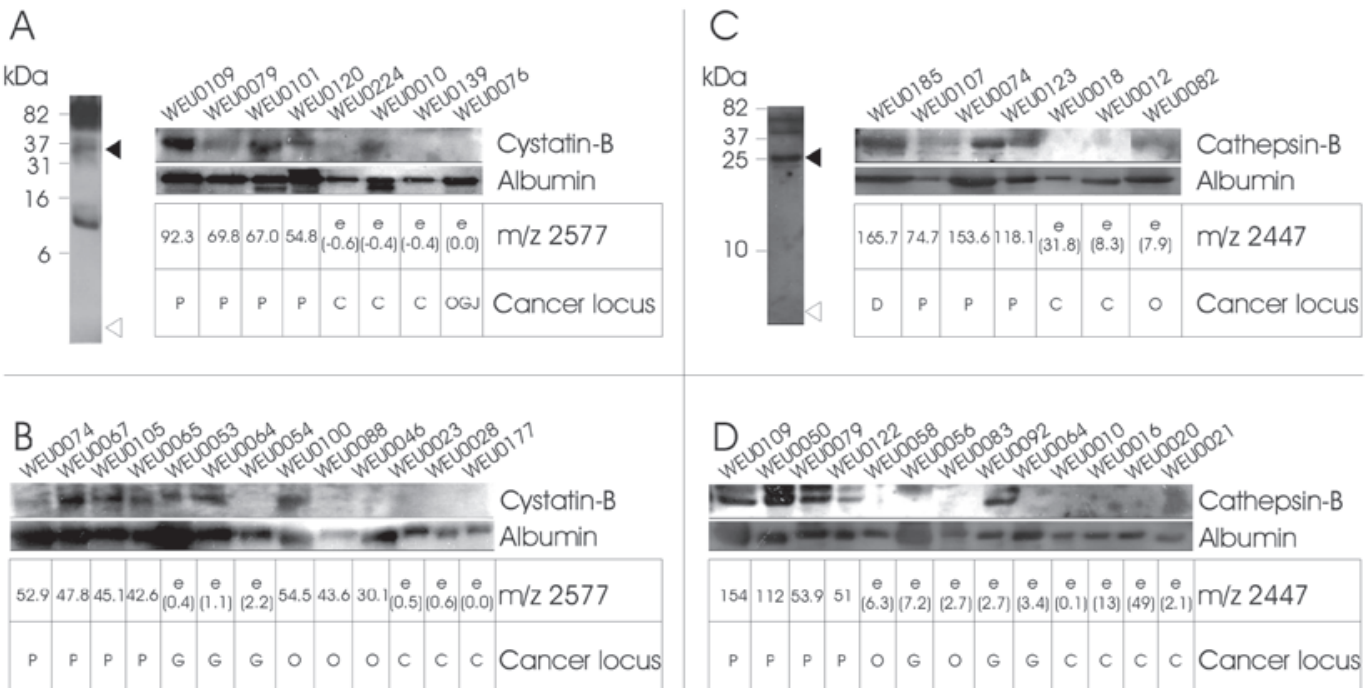

Figure 2. Validation of identified proteins by western blotting. Urine samples were separated by $20 \%$ SDS-PAGE and analysed by western blotting using antibodies specific against CSTB, CTSB and albumin. Panels (A) and (C) (left) show the western blotting results of combined urine samples in the 1-100-kDa range. The samples were tested initially for the presence of fragments of CSTB and CTSB in the region of the measured $\mathrm{m} / \mathrm{z}$ (open triangles), as well as full-length molecules (filled triangles) or other breakdown products. Reliable signals in the 35-kDa range for CSTB and 25-kDa for CTSB were then further analysed. Validation and confirmation of LC-MS/MS and Mascot results are shown in the strip-blots in panels (A) and (C), which show the results of (A) 8 or (C) 7 urine samples that were used in LC-MS/MS and subsequent Mascot searches, together with the cluster peak intensity matrices derived from the SELDI analysis (underneath the individual blots). Panels (B) and (D) depict the analysis of four random cancer and four control samples. The samples were selected based on the IMAC30 SELDI analysis for the presence of a peak cluster at m/z 2577 for CSTB, and on the CM10 SELDI peak pattern at m/z 2447 for CTSB (cancer samples) or the absence of these $\mathrm{m} / \mathrm{z}$ peaks (healthy controls). 'e' corresponds to an estimated value (in brackets), where no peak at the $\mathrm{m} / \mathrm{z}$ point could be detected above the $\mathrm{S} / \mathrm{N}$ ratio. Qualitative loading control was performed by probing against the common urinary molecule serum albumin. Molecular weight indicators are depicted on the left, and the target proteins on the right of the blots. The cancer locus is the histologically assessed cancer site of duodenum (D), pancreas (P), oesophagogstric junction (OGJ), oesophagus (O), or non-cancerous control (C). CTSB, cathepsin-B; CSTB, cystatin-B; SELDI, surface-enhanced laser desorption/ionization; MS, mass spectrometry; $\mathrm{S} / \mathrm{N}$, signal-to-noise ratio.

Development of a cancer profiling database. Over the last decade, the emergence of high-throughput screening platforms opened the possibility to mechanistically characterize at the molecular level disease phenotypes, disease subtypes, assess disease progression and monitor response to therapy. However, despite a crescendo in the availability of these large-scale multi-omics data for numerous conditions, no apparent or adequate effort has been made to curate and integrate those data resources.

Therefore, large-scale multi-omics data handling differential expression of several molecular entities, for example, microRNA, gene, protein and metabolite across multiple tissues and biological fluids in several cancer types (with special emphasis on GI neoplasms) were collected from the literature and general scope databases, and subjected to extensive manual curation and annotation. This effort yielded a cancer profiling database: the Multi-Omics Cancer database MoCadb (www.padb.org/mocadb), a database module of the Pan-Omics Analysis Database (PADB) (www.PADB.org) which ensures a long-term lifecycle of the created repository, along with providing the appropriate framework that encloses curated resources, in order to assist in the development of integrative -omics disease models, straightforward translation of findings from experimental animal models using established ortholog maps, creation of cellular regulatory networks based on the association of miRNAs and transcription factors to targets, protein-protein and gene-gene interactions, enzymatic reactions, delineation of pathways and drug interactions.

MoCadb aims to incorporate existing molecular information and clinical metadata, ultimately holding the potential to unravel and allow an in-depth understanding of the regulation of key molecules modulating pathophysiology and progression in several cancer types.

\section{Discussion}

The identification of potential novel biomarkers for ailments such as pancreatic cancer is of utmost importance, particularly in light of the shortcomings of the markers currently used in the clinical setting for diagnosis and disease progression monitoring. As an example, in a retrospective study, cohort screening using a bead-based antibody array of a multi-parametric signature of three serum biomarkers, namely CA19.9, intercellular adhesion molecules 1 and osteoprotegerin, were able to distinguish patients with pancreatic ductal adenocarcinoma (PDAC) from healthy subjects, with a sensitivity/specificity of $88 / 90 \%$ and an area under the curve (AUC) of 0.93 (38). However, in a follow-up study using a larger prospective cohort, the latter biomarker panel and the respective AUCs associated with the biomarker model were not statistically different, thereby failing to distinguish PDAC cases from matched controls (39). This highlights the current issues and challenges associated with biomarker research in pre-diagnostic risk assessment of pancreatic cancer.

We selected an MS-based proteomic screening of urine from upper GI cancer patients for prospective biomarkers for the four most prominent cancer types associated with the upper GI to establish a proteomic fingerprint pattern, as well as defined molecular markers, which can potentially be used in clinical diagnostics. In the clinical setting, urine is an optimal sample source, as it is easy to obtain, the collection is non-invasive, and is relatively stable in terms of sample 
integrity. A substantial number of previous studies have found the SELDI-TOF-MS technique ideally suited for urine analysis, with a combination of high throughput, speed and relatively low cost (40-42). However, a main drawback of this technique is the comparatively medium resolution of the spectra obtained, but this is adequate to resolve peaks in the $1-25-\mathrm{kDa}$ range from spectra with $<500$ peaks. We have previously demonstrated that both IMAC30 and CM10 are useful chip types for the analysis of human urine (17), and we were able to generate a decision-tree model for upper GI cancer with an overall sensitivity of $98 \%$ and a specificity of $87 \%$. In the present study, we identified 8 peak clusters by SELDI-TOF-MS for the four cancer types studied and, using expression pattern matching, we were able to assign several proteins identified in the urine to our proposed biomarkers.

An elevated amount of SERPINA3 may be associated with both gastric and pancreatic cancer, and has also been reported as a potential urinary biomarker in non-small-cell lung cancer (43). SERPINA3 is a protease inhibitor that can modulate cathepsin G (44), and its glycosylated form is directly associated with colorectal cancer (45). This protein was already known to be associated with pancreatic cancer and could be detected in higher levels in the plasma $(46,47)$. Furthermore, this marker was specifically negatively correlated with survival in patients with advanced pancreatic cancer (48). However, it is also worth noting that several different fragments from the same molecule were observed in our SELDI-TOF experiments, namely m/z 2444, 2447 and 4639, of which the latter is specific for gastric cancer. Additionally, we also identified the same molecule in our upper GI cancer screen (17) as m/z 8803. It is possible that a specific proteolytic fragment of SERPINA3 is associated with and generated in a specific type of cancer, where the $\mathrm{m} / \mathrm{z} 8803$ fragment may be generic for upper GI cancer and the small fragments specific to pancreatic cancer. This is further substantiated by our observation that the protease CTSB is also a potential biomarker for pancreatic cancer. Additionally, CTSB was validated as a potential urinary biomarker in pancreatic carcinoma by western blotting. Furthermore, CTSB was reported to be a prognostic marker in pancreatic adenocarcinoma (49), which confirms our findings for this molecule and its potential as a lead marker in pancreatic cancer diagnostics. Another promising candidate as a pancreatic cancer biomarker was identified and verified as CSTB. The cysteine protease inhibitor CSTB was described as a protein that is able to stimulate cancer cell growth in vitro and in vivo (50). Other potential pancreatic cancer markers identified in this study comprise fragments of immunoglobulins. The occurrence of specific fragments of antibodies may be associated with the increased amounts of CTSB, or may be due to a host response to pancreatic tumour growth. Antibodies are also well-described and used in the clinical setting to assess various cancer types (e.g., CA19-9 in pancreatic cancer) $(9,10)$.

Stratification of OGJ cancer cases by SELDI-TOF-MS revealed two potential m/z peak clusters, m/z 4908 and 5511 . The latter peak cluster was identified to be a fragment of PDCD6IP (also referred to as AIP1 or ALIX), which has been described to participate in programmed cell death, and it was reported that its overexpression can block apoptosis (51). The m/z 4908 peak cluster consists of fragments from VMO1 and TPI. No role of VMO1 has been implicated in cancer, and this may be a novel target for OGJ cancer, whereas TPI was described in the literature to be upregulated in oesophageal cancer (52), as well as in hepatocellular carcinoma (53).

The oesophageal cancer marker of $\mathrm{m} / \mathrm{z} 4141$ appears to contain several molecular constituents, namely C4A, ACPP, AZU1 and fragments from Histone H1. C4A is an important component in the activation of the classical pathway of the complement system and proteolytic breakdown products of C4-A have been suggested as biomarkers in breast cancer (54), although a specific proteolytic product, $\mathrm{C} 4 \mathrm{a}$ anaphylatoxin, is a mediator of local inflammatory processes (55). This protein is therefore potentially unsuitable as a diagnostic marker in oesophageal cancer. ACPP, a non-specific tyrosine phosphatase, is well-described to be associated with prostate cancer (56), and is used clinically as a diagnostic marker. AZU1, an antibacterial and monocyte- and fibroblast-specific chemotactic glycoprotein, which acts in conjunction with cathepsin $\mathrm{G}$ in host-defense mechanisms (57), was hypothesized to be a potential pancreatic cancer biomarker in the pancreatic juice (58). Histones $\mathrm{H} 1$ have been reported to be involved in the survival of breast cancer cells (59), and H1.2 specifically was identified as an apoptogenic factor (60).

In conclusion, the approach of using SELDI-MS to identify potential lead candidates as biomarkers associated with specific upper GI cancers is a useful tool that enabled us to identify potential global upper GI cancer markers, as well as potentially specific markers for individual cancer types, such as gastric, pancreatic, OGJ and oesophageal cancer. CSTB and CTSB, in particular, appear to be promising lead candidates, since these molecules are not commonly found in the urine, and have already been associated with pancreatic cancer in situ in the literature (61-63). Other potential lead markers may require further validation, such as western blotting and, ultimately, exact determination of the sensitivity/specificity values of our novel proposed markers associated with specific disease states and their usefulness in a more general setting. Further studies, including an extended cohort, will help determine the validity of our findings, and specific assays monitoring the expression levels of our proposed biomarkers will help to translate our findings into the clinical setting. One of the main hurdles to overcome is a methodological/technical dependency, such as the use of the SELDI technology, which is not straightforward to translate into a clinical environment. A more appropriate approach would rely on techniques that are commonly in use, such as specific protein detection methods. Additional investigations are needed to explain the biological involvement of the proposed biomarkers in tissue type-specific cancers and their presence in the urine. To aid in this task, and to add a higher level of contextualisation of individual markers or a panel of biomolecules, information held in our MoCadb database, and in other databases within the PADB initiative, can guide the biomarker identification process. Future expansion of this resource, by also incorporating large-scale datasets derived from other cancer tissue type studies, will allow us to not only investigate the biological relevance, importance and usefulness of individual biomolecules, but also to identify potential intervention points or markers of high prognostic/diagnostic value prior to evaluation and validation in patient cohorts. Potential applications of such 
findings will most likely include the use of multiplexed platforms, since it was shown that integrating various molecule types, such as proteins and miRs, can exert a beneficial effect on overall sensitivity and specificity of a bioassay for pancreatic cancer (64). However, this also emphasises that further study is required to use novel approaches and may lead to better outcomes in patient stratification and disease treatment.

\section{Acknowledgements}

The authors would like to thank N.A. Stephens for patient recruitment and J. Black for technical assistance in western blotting.

\section{Funding}

The present study was supported by the University of Edinburgh and the European Union's Seventh Framework Programme FP7/2007-2013 under grant agreement FP7-PEOPLE-2013-ITN-608332.

\section{Authors' contributions}

HH performed the sample preparations, SELDI measurements, data analysis, Mascot searches, software design and coding, and wrote the manuscript; ADC performed the LC-MS/MS measurements; MF co-wrote the manuscript and developed MoCadb; RJS and JM co-wrote the manuscript; JAR and KCHF supervised the research.

\section{Availability of data and materials}

All the datasets generated and analysed in the present study are included in this published manuscript.

\section{Ethics approval and consent to participate}

All procedures were approved by the local research ethics committee and written informed consent was obtained from the patients. The study conformed to the standards set by the Declaration of Helsinki.

\section{Patient consent for publication}

Not applicable.

\section{Competing interests}

The authors declare that they have no competing interests.

\section{References}

1. Ajani JA, D'Amico TA, Almhanna K, Bentrem DJ, Besh S, Chao J, Das P, Denlinger C, Fanta P, Fuchs CS, et al; National comprehensive cancer network: Esophageal and esophagogastric junction cancers, version 1.2015. J Natl Compr Canc Netw 13: 194-227, 2015

2. Fitzmaurice C, Dicker D, Pain A, Hamavid H, Moradi-Lakeh M, MacIntyre MF, Allen C, Hansen G, Woodbrook R, Wolfe C, et al Global Burden of Disease Cancer Collaboration: The Global Burden of Cancer 2013. JAMA Oncol 1: 505-527, 2015.
3. De Angelis R, Sant M, Coleman MP, Francisci S, Baili P, Pierannunzio D, Trama A, Visser O, Brenner H, Ardanaz E, et al; EUROCARE-5 Working Group: Cancer survival in Europe 1999-2007 by country and age: Results of EUROCARE--5-a population-based study. Lancet Oncol 15: 23-34, 2014.

4. Lagergren J, Smyth E, Cunningham D and Lagergren P: Oesophageal cancer. Lancet 390: 2383-2396, 2017.

5. Kamisawa T, Wood LD, Itoi T and Takaori K: Pancreatic cancer. Lancet 388: 73-85, 2016.

6. Kim MI: Surprised by tears. Christ Nurse Int 5: 6-7, 1989.

7. Grote T, Siwak DR, Fritsche HA, Joy C, Mills GB, Simeone D, Whitcomb DC and Logsdon CD: Validation of reverse phase protein array for practical screening of potential biomarkers in serum and plasma: Accurate detection of CA19-9 levels in pancreatic cancer. Proteomics 8: 3051-3060, 2008.

8. Lee G, Ge B, Huang TK, Zheng G, Duan J and Wang IH: Positive identification of CA215 pan cancer biomarker from serum specimens of cancer patients. Cancer Biomark 6: 111-117, 2010.

9. Lee G, Laflamme E, Chien $\mathrm{CH}$ and Ting $\mathrm{HH}$ : Molecular identity of a pan cancer marker, CA215. Cancer Biol Ther 7: 2007-2014, 2008.

10. Zhang S, Wang YM, Sun CD, Lu Y and Wu LQ: Clinical value of serum CA19-9 levels in evaluating resectability of pancreatic carcinoma. World J Gastroenterol 14: 3750-3753, 2008.

11. Kim JE, Lee KT, Lee JK, Paik SW, Rhee JC and Choi KW: Clinical usefulness of carbohydrate antigen 19-9 as a screening test for pancreatic cancer in an asymptomatic population. J Gastroenterol Hepatol 19: 182-186, 2004.

12. Singh S, Tang SJ, Sreenarasimhaiah J, Lara LF and Siddiqui A: The clinical utility and limitations of serum carbohydrate antigen (CA19-9) as a diagnostic tool for pancreatic cancer and cholangiocarcinoma. Dig Dis Sci 56: 2491-2496, 2011.

13. Lee G: Cancer cell-expressed immunoglobulins: CA215 as a pan cancer marker and its diagnostic applications. Cancer Biomark 5: 137-142, 2009.

14. Hao Y, Yu Y, Wang L, Yan M, Ji J, Qu Y, Zhang J, Liu B and Zhu Z: IPO-38 is identified as a novel serum biomarker of gastric cancer based on clinical proteomics technology. J Proteome Res 7: 3668-3677, 2008.

15. Hardt PD and Ewald N: Tumor M2 pyruvate kinase: A tumor marker and its clinical application in gastrointestinal malignancy. Expert Rev Mol Diagn 8: 579-585, 2008.

16. Gaber A, Johansson M, Stenman UH, Hotakainen K, Pontén F, Glimelius B, Bjartell A, Jirström K and Birgisson H: High expression of tumour-associated trypsin inhibitor correlates with liver metastasis and poor prognosis in colorectal cancer. Br J Cancer 100: 1540-1548, 2009.

17. Husi H, Stephens N, Cronshaw A, MacDonald A, Gallagher I, Greig C, Fearon KC and Ross JA: Proteomic analysis of urinary upper gastrointestinal cancer markers. Proteomics Clin Appl 5: 289-299, 2011.

18. Sofiadis A, Dinets A, Orre LM, Branca RM, Juhlin CC, Foukakis T, Wallin G, Höög A, Hulchiy M, Zedenius J, Larsson C and Lehtiö J: Proteomic study of thyroid tumors reveals frequent up-regulation of the $\mathrm{Ca}^{2+}$-binding protein S100A6 in papillary thyroid carcinoma. Thyroid 20: 1067-1076, 2010.

19. Wang XH, Zhang LH, Zhong XY, Xing XF, Liu YQ, Niu ZJ, Peng Y, Du H, Zhang GG, Hu Y, et al: S100A6 overexpression is associated with poor prognosis and is epigenetically up-regulated in gastric cancer. Am J Pathol 177: 586-597, 2010.

20. Wei BR, Hoover SB, Ross MM, Zhou W, Meani F, Edwards JB, Spehalski EI, Risinger JI, Alvord WG, Quiñones OA, et al: Serum S100A6 concentration predicts peritoneal tumor burden in mice with epithelial ovarian cancer and is associated with advanced stage in patients. PLoS One 4: e7670, 2009.

21. Hua Z, Chen J, Sun B, Zhao G, Zhang Y, Fong Y, Jia Z and Yao L Specific expression of osteopontin and S100A6 in hepatocellular carcinoma. Surgery 149: 783-791, 2011.

22. Komatsu K, Andoh A, Ishiguro S, Suzuki N, Hunai H, Kobune-Fujiwara Y, Kameyama M, Miyoshi J, Akedo H and Nakamura H: Increased expression of S100A6 (calcyclin), a calcium-binding protein of the S100 family, in human colorectal adenocarcinomas. Clin Cancer Res 6: 172-177, 2000.

23. Sanders ME, Dias EC, Xu BJ, Mobley JA, Billheimer D, Roder H, Grigorieva J, Dowsett M, Arteaga CL and Caprioli RM: Differentiating proteomic biomarkers in breast cancer by laser capture microdissection and MALDI MS. J Proteome Res 7: 1500-1507, 2008. 
24. Loos M, Bergmann F, Bauer A, Hoheisel JD, Esposito I, Kleeff J, Schirmacher P, Büchler MW, Klöppel G and Friess H: Solid type clear cell carcinoma of the pancreas: differential diagnosis of an unusual case and review of the literature. Virchows Archiv 450: 719-726, 2007.

25. Fry LC, Mönkemüller K and Malfertheiner P: Molecular markers of pancreatic cancer: Development and clinical relevance. Langenbecks Arch Surg 393: 883-890, 2008

26. Ljungberg B: Prognostic markers in renal cell carcinoma. Curr Opin Urol 17: 303-308, 2007.

27. Higgins JP: Gene array studies in renal neoplasia. ScientificWorldJournal 6: 502-511, 2006.

28. Wang J, Chen J and Sen S: MicroRNA as Biomarkers and Diagnostics. J Cell Physiol 231: 25-30, 2016.

29. Lindner K, Haier J, Wang Z, Watson DI, Hussey DJ and Hummel R: Circulating microRNAs: emerging biomarkers for diagnosis and prognosis in patients with gastrointestinal cancers. Clin Sci (Lond) 128: 1-15, 2015.

30. Schultz NA, Dehlendorff C, Jensen BV, Bjerregaard JK, Nielsen KR, Bojesen SE, Calatayud D, Nielsen SE, Yilmaz M, Holländer NH, et al: MicroRNA biomarkers in whole blood for detection of pancreatic cancer. JAMA 311: 392-404, 2014.

31. Bosco C, Wulaningsih W, Melvin J, Santaolalla A, De Piano M, Arthur R and Van Hemelrijck M: Metabolic serum biomarkers for the prediction of cancer: A follow-up of the studies conducted in the Swedish AMORIS study. Ecancermedicalscience 9: 555, 2015.

32. Xie G, Lu L, Qiu Y, Ni Q, Zhang W, Gao YT, Risch HA, Yu H and Jia W: Plasma metabolite biomarkers for the detection of pancreatic cancer. J Proteome Res 14: 1195-1202, 2015.

33. Hirata Y, Kobayashi T, Nishiumi S, Yamanaka K, Nakagawa T, Fujigaki S, Iemoto T, Kobayashi M, Okusaka T, Nakamori S, et al: Identification of highly sensitive biomarkers that can aid the early detection of pancreatic cancer using GC/MS/MS-based targeted metabolomics. Clin Chim Acta 468: 98-104, 2017.

34. Husi H, Skipworth RJ, Cronshaw A, Fearon KC and Ross JA: Proteomic identification of potential cancer markers in human urine using subtractive analysis. Int J Oncol 48: 1921-1932, 2016.

35. Kalantari S, Jafari A, Moradpoor R, Ghasemi E and Khalkhal E: Human Urine Proteomics: Analytical Techniques and Clinical Applications in Renal Diseases. Int J Proteomics 2015: 782798, 2015.

36. Wu J, Chen YD and Gu W: Urinary proteomics as a novel tool for biomarker discovery in kidney diseases. J Zhejiang Univ Sci B 11: 227-237, 2010.

37. Husi H, Ward MA, Choudhary JS, Blackstock WP and Grant SG: Proteomic analysis of NMDA receptor-adhesion protein signaling complexes. Nat Neurosci 3: 661-669, 2000.

38. Brand RE, Nolen BM, Zeh HJ Allen PJ, Eloubeidi MA, Goldberg M, Elton E, Arnoletti JP, Christein JD, Vickers SM, et al: Serum biomarker panels for the detection of pancreatic cancer. Clin Cancer Research 17: 805-816, 2011.

39. Nolen BM, Brand RE, Prosser D, Velikokhatnaya L, Allen PJ, Zeh HJ, Grizzle WE, Huang Y, Lomakin A and Lokshin AE: Prediagnostic serum biomarkers as early detection tools for pancreatic cancer in a large prospective cohort study. PLoS One 9: e94928, 2014

40. Caffrey RE: A review of experimental design best practices for proteomics based biomarker discovery: Focus on SELDI-TOF. Methods Mol Biol 641: 167-183, 2010.

41. Okamoto A, Yamamoto H, Imai A, Hatakeyama S, Iwabuchi I, Yoneyama T, Hashimoto Y, Koie T, Kamimura N, Mori K, et al: Protein profiling of post-prostatic massage urine specimens by surface-enhanced laser desorption/ionization time-of-flight mass spectrometry to discriminate between prostate cancer and benign lesions. Oncol Rep 21: 73-79, 2009.

42. Gagnon A, Shi Q and Ye B: Surface-enhanced laser desorption/ionization mass spectrometry for protein and Peptide profiling of body fluids. Methods Mol Biol 441: 41-56, 2008.

43. Zhang Y, Li Y, Qiu F and Qiu Z: Comparative analysis of the human urinary proteome by 1D SDS-PAGE and chip-HPLC-MS/MS identification of the AACT putative urinary biomarker. J Chromatogr B Analyt Technol Biomed Life Sci 878: 3395-3401, 2010.

44. Rubin H, Wang ZM, Nickbarg EB, McLarney S, Naidoo N, Schoenberger OL, Johnson JL and Cooperman BS: Cloning, expression, purification, and biological activity of recombinant native and variant human alpha 1-antichymotrypsins. J Biol Chem 265: 1199-1207, 1990.
45. Pinczower GD, Williams RP, Gianello RD, Robinson HC, Preston BN and Linnane AW: Characterisation of the tumour-associated carbohydrate epitope recognised by monoclonal antibody 4D3. Int J Cancer 66: 636-644, 1996.

46. Koomen JM, Shih LN, Coombes KR, Li D, Xiao LC, Fidler IJ, Abbruzzese JL and Kobayashi R: Plasma protein profiling for diagnosis of pancreatic cancer reveals the presence of host response proteins. Clin Cancer Res 11: 1110-1118, 2005.

47. $\mathrm{Yu}$ KH, Rustgi AK and Blair IA: Characterization of proteins in human pancreatic cancer serum using differential gel electrophoresis and tandem mass spectrometry. J Proteome Res 4: $1742-1751,2005$

48. Roberts AS, Campa MJ, Gottlin EB, Jiang C, Owzar K, Kindler HL, Venook AP, Goldberg RM, O'Reilly EM and Patz EF Jr: Identification of potential prognostic biomarkers in patients with untreated, advanced pancreatic cancer from a phase 3 trial (Cancer and Leukemia Group B 80303). Cancer 118: 571-578, 2012.

49. Niedergethmann M, Wostbrock B, Sturm JW, Willeke F, Post S and Hildenbrand R: Prognostic impact of cysteine proteases cathepsin B and cathepsin $\mathrm{L}$ in pancreatic adenocarcinoma. Pancreas 29: 204-211, 2004

50. Hosokawa M, Kashiwaya K, Eguchi H, Ohigashi H, Ishikawa O, Furihata M, Shinomura Y, Imai K, Nakamura Y and Nakagawa H: Over-expression of cysteine proteinase inhibitor cystatin 6 promotes pancreatic cancer growth. Cancer Sci 99: 1626-1632, 2008.

51. Wu Y, Pan S, Che S, He G, Nelman-Gonzalez M, Weil MM and Kuang J: Overexpression of $\mathrm{Hp} 95$ induces G1 phase arrest in confluent HeLa cells. Differentiation 67: 139-153, 2001.

52. Huang ZY, Xiong G, Zhang J and Wang WJ: [Screening of differentially expressed proteins from human esophageal cancer and esophageal tissues by two-dimensional difference gel electrophoresis and mass spectrometry]. Nan Fang Yi Ke Da Xue Xue Bao 27: 1406-1409, 2007 (In Chinese).

53. Hamaguchi T, Iizuka N, Tsunedomi R, Hamamoto Y, Miyamoto T, Iida M, Tokuhisa Y, Sakamoto K, Takashima M, Tamesa T and Oka M: Glycolysis module activated by hypoxia-inducible factor 1alpha is related to the aggressive phenotype of hepatocellular carcinoma. Int J Oncol 33: 725-731, 2008

54. van den Broek I, Sparidans RW, Schellens JH and Beijnen JH: Quantitative assay for six potential breast cancer biomarker peptides in human serum by liquid chromatography coupled to tandem mass spectrometry. J Chromatogr B Analyt Technol Biomed Life Sci 878: 590-602, 2010

55. Hack CE, Nuijens JH, Felt-Bersma RJ, Schreuder WO, Eerenberg-Belmer AJ, Paardekooper J, Bronsveld W and Thijs LG: Elevated plasma levels of the anaphylatoxins C3a and $\mathrm{C} 4 \mathrm{a}$ are associated with a fatal outcome in sepsis. Am J Med 86: 20-26, 1989.

56. Hassan MI, Aijaz A and Ahmad F: Structural and functional analysis of human prostatic acid phosphatase. Expert Rev Anticancer Ther 10: 1055-1068, 2010.

57. Miyasaki KT and Bodeau AL: Human neutrophil azurocidin synergizes with leukocyte elastase and cathepsin G in the killing of Capnocytophaga sputigena. Infect Immun 60: 4973-4975, 1992.

58. Grønborg M, Bunkenborg J, Kristiansen TZ, Jensen ON, Yeo CJ, Hruban RH, Maitra A, Goggins MG and Pandey A: Comprehensive proteomic analysis of human pancreatic juice. $\mathrm{J}$ Proteome Res 3: 1042-1055, 2004.

59. Sancho M, Diani E, Beato M and Jordan A: Depletion of human histone $\mathrm{H} 1$ variants uncovers specific roles in gene expression and cell growth. PLoS Genet 4: e1000227, 2008.

60. Zong WX: Histone, H1.2: Another housekeeping protein that kills. Cancer Biol Ther 3: 42-43, 2004.

61. Chen S, Dong H, Yang S and Guo H: Cathepsins in digestive cancers. Oncotarget 8: 41690-41700, 2017.

62. Aggarwal N and Sloane BF: Cathepsin B: Multiple roles in cancer. Proteomics Clin Appl 8: 427-437, 2014.

63. Gondi CS and Rao JS: Cathepsin B as a cancer target. Expert Opin Ther Targets 17: 281-291, 2013

64. Madhavan B, Yue S, Galli U, Rana S, Gross W, Müller M, Giese NA, Kalthoff H, Becker T, Büchler MW and Zöller M: Combined evaluation of a panel of protein and miRNA serum-exosome biomarkers for pancreatic cancer diagnosis increases sensitivity and specificity. Int J Cancer 136: 2616-2627, 2015. 